\title{
Uma Metodologia para Análise da Distribuição de Contagem dos Picos de Tensão de Reignição para Avaliação da Estabilidade do Processo GMAW Curto-Circuito
}

Guilherme Rezende Bessa Ferreira ${ }^{1}$, Rafaela Abreu Campos ${ }^{1}$, Roberto Malheiros Moreira Filho ${ }^{1}$, Américo Scotti ${ }^{2}$, Moisés Luiz Lagares Jr ${ }^{1}$ (])

${ }^{1}$ Universidade Federal de Juiz de Fora - UFJF, Laboratório para Otimização de Processos de Soldagem, Juiz de Fora, MG, Brasil.

${ }^{2}$ Universidade Federal de Uberlândia - UFU, Grupo Laprosolda, Centro para Pesquisa e Desenvolvimento de Processos de Soldagem, Uberlândia, MG, Brasil.

Como citar: Ferreira GRB, Campos RA, Moreira Filho RM, Scotti A, Lagares Jr ML. Uma metodologia para análise da distribuição de contagem dos picos de tensão de reignição para avaliação da estabilidade do processo GMAW curto-circuito. Soldagem \& Inspeção. 2019;24:e2407. https://doi.org/10.1590/0104-9224/SI24.07

Resumo: A estabilidade do arco tem sido estudada através de análises estatísticas obtidas dos sinais de tensão de arco e corrente de soldagem. Histogramas e cálculo de estatísticas dos parâmetros tempo de arco aberto e tempo de curto circuito são meios de avaliação da estabilidade dos processos de soldagem. Contudo, estudos relacionados aos picos de tensão de reignição do arco elétrico ainda são pouco explorados. Desta forma, este trabalho apresenta uma metodologia de avaliação da distribuição de picos de reignição do arco do processo Gas Metal Arc Welding por curtocircuito (GMAW-CC). Cordões de solda depositados em chapa plana, variando o gás, foram executados a fim de produzir diferentes distribuições de picos de reignição. $O$ teste qui-quadrado foi aplicado para avaliar a distribuição de frequências observadas de picos de tensão e compará-la com a distribuição hipotética. Um cordão padrão foi comparado com sua repetição e testada a hipótese de distribuições iguais. Os resultados mostraram que existem distribuições estatisticamente iguais de picos de tensão entre cordões de solda. Concluiu-se que a metodologia proposta permitiu o estudo da distribuição de picos de reignição. Também foi demonstrada haver potencialmente uma outra forma de avaliar a estabilidade de arcos em soldagem GMAW-CC, através do teste $\chi 2$.

Palavras-chave: Processo de soldagem; Estabilidade; GMAW Curto-Circuito; Reignição do arco.

\section{A Methodology for the Counting Distribution Analysis of the Reignition Voltage Peaks for Stability Evaluation of Short Circuit GMAW Process}

\begin{abstract}
The stability of the welding arc has been studied through statistical analysis, obtained mainly from the voltage and current signals. Histograms and statistics of open arc time and short circuit time are important means of evaluating the welding processes stability. However, studies related to electric arc reignition voltage peaks are still poorly explored. Thus, this work presents a methodology for the evaluation of the arc re-ignition voltage peaks of the Short-Circuit Gas Metal Arc Welding (GMAW-SC) process and its relation with the regularity of the welding voltage signals. Weld beads were deposited on flat plate, varying the gas, in order to produce different distributions of re-ignition peaks. Pearson's chi-square test was applied to evaluate the distribution of peak voltage frequencies and compared with the hypothetical distribution. A standard weld bead was compared to its replication and tested for equal distributions. The results showed that exist equal voltage peaks distributions between weld beads, being dependent on the welding parameters. It was concluded that the proposed methodology is able to the statistical study of the distribution of welding arc re-ignition peaks. It was also demonstrated that the arc stability in GMAW-SC can be evaluated by the analysis of the voltage peaks distribution.
\end{abstract}

Key-words: Welding Processes; Stability; Short-circuit GMAW process; Arc re-ignition.

\section{Introdução}

A soldagem é um processo físico-químico complexo e sua estabilidade está intimamente relacionada com a transferência metálica. A estabilidade dos processos de soldagem a arco elétrico tem sido avaliada por diferentes métodos [1]. Emissão acústica [2], avaliação dinâmica via ciclogramas [3], decomposição de sinais [4] e aplicação do expoente de Lyapunov [5] são exemplos de métodos aplicados ao estudo da estabilidade. Outros métodos adotam a avaliação dos sinais de corrente de soldagem e tensão do arco, tendo a estatística um importante papel como ferramenta de análise [6-8]. Tais métodos são especialmente adequados ao estudo do processo Gas Metal Arc Welding por curto-circuito (GMAW-CC), sendo encontrada ampla literatura relacionando os tempos de curto circuito e arco aberto com a regularidade do processo. 
Suban e Tusek [1] afirmam que a estabilidade do processo GMAW-CC é uma propriedade do arco de soldagem e um arco ideal deveria apresentar uniformidade nos tempos de arco aberto e tempos de curto-circuito. Liu e Siewert [9] confirmam que a regularidade do processo GMAW-CC pode ser estudada pela avaliação da variância do período de transferência da gota metálica. Em estudo recente de Chen et al. [10], a regularidade do processo GMAW com transferência CMT (cold metal transfer) também foi avaliada pela variância do período de curto-circuito. Hermans and Ouden [11] concluíram que a máxima estabilidade do processo ocorre quando o desvio padrão da frequência de transferência da gota é mínimo. Baseado na análise de resposta em frequência, Chu et al. [12] concluíram que um processo estável, com cordões de solda uniformes, pode ser obtido quando a frequência de curto-circuito atinge um valor máximo no domínio da tensão de soldagem e se mantém estável. Souza et al. [13] obtiveram resultados semelhantes. Kang e Rhee [14] concluíram que soldagens com tensões acima ou abaixo de valores ótimos torna o arco instável e grande quantidade de respingos é gerada.

Por outro lado, Zhu et al. [15] investigaram a natureza da variação do tamanho da gota metálica e seu efeito no comportamento do processo GMAW-CC. Os resultados experimentais revelaram natureza aleatória da distribuição do tamanho da gota (medida no momento anterior ao curto-circuito) e que uma das origens desse comportamento é a aleatoriedade da energia do arco. Essa é uma importante constatação pois mostra que não somente a regularidade da frequência de curto circuito deve ser avaliada mas também a transição do curto circuito para o arco aberto (reignição do arco). Não obstante, a tensão de reignição do arco é outro possível parâmetro de avaliação da estabilidade pois está ligada ao grau de dificuldade de reignição do arco no processo GMAW-CC [16]. Contudo, poucos são os trabalhos neste sentido, destacando-se Scotti et al. [17] que propôs um índice que mede a estabilidade do processo através do cálculo da potência elétrica gasta para reignição do arco após o curto circuito.

Desta forma, este trabalho objetiva propor uma metodologia alternativa para avaliação da estabilidade do arco em soldagem GMAW-CC através da avaliação da distribuição de picos de tensão de reignição do arco e sua relação com a regularidade dos sinais de tensão de soldagem.

\section{Procedimento Experimental}

O processo utilizado foi o GMAW-CC com avanço mecanizado da tocha de soldagem. Os experimentos foram realizados na forma de simples deposição (sem junta) sobre placas de teste de aço carbono ABNT 1020, de 3,2 mm de espessura e $38,1 \mathrm{~mm}$ de largura. Limpeza prévia das chapas foi feita, com retirada de carepas de laminação e lavagem com álcool absoluto para eliminação de poeira e filmes de óleo. Foi utilizada uma fonte comercial inversora no secundário, com os parâmetros fixos de soldagem mostrados na Tabela 1. O metal de adição utilizado foi o AWS ER70S-6 (carretel novo), 1,0 mm de diâmetro. Os gases de proteção utilizados foram argônio puro, mistura $\mathrm{Ar}+20 \% \mathrm{CO}_{2}$ e $\mathrm{CO}_{2}$ puro, todos a uma vazão de $16 \mathrm{l} / \mathrm{min}$ e diâmetro de bocal da tocha de $16,0 \mathrm{~mm}$. Todos os cordões de solda foram monitorados em tempo real para garantia de fornecimento constante de fluxo de gás e alimentação de arame através de sistema dedicado de aquisição de dados SAP-4 (marca IMC soldagem). O mesmo sistema foi usado para aquisição dos valores de tensão de arco e corrente de soldagem a uma taxa de aquisição de $5.000 \mathrm{~Hz}$.

Tabela 1. Parâmetros de soldagem usados em todos os experimentos.

\begin{tabular}{cc}
\hline Uref $_{\text {r Tensão de Referência (V) }}$ & $\mathbf{1 8}$ \\
Velocidade real de alimentação $(\mathrm{mm} / \mathrm{s})$ & 50,0 \\
Velocidade de soldagem $(\mathrm{mm} / \mathrm{s})$ & 5,0 \\
Distância de bico de contato à peça $(\mathrm{mm})$ & 12 \\
Ângulo entre a tocha e a superfície soldada & 900 \\
Posição de indutância* de subida e de descida da máquina de solda & 30 \\
Posição de soldagem & plana \\
\hline
\end{tabular}

*para máquina de solda DIGIPLUS A7 450, marca IMC soldagem.

\section{Metodologia para Determinação da Distribuição de Picos de Tensão em Soldagem Padrão GMAW-CC}

A soldagem GMAW-CC convencional se caracteriza por uma sequência de curtos-circuitos entre a ponta do eletrodo e a poça de fusão, como ilustra a Figura 1. Estes curtos acontecem pois, de forma transitória, a taxa de fusão do arame se torna menor do que sua taxa de alimentação. Ao acontecer o curto-circuito, a tensão cai drasticamente (a resistência entre arame e poça é pequena). Durante o curto-circuito, a gota em formação na ponta do arame é sugada pela poça por tensão superficial e, por estar em curto, concomitantemente a corrente progressivamente cresce, facilitando sua transferência (efeito pinch). Como durante o curto-circuito o arco está apagado, o aquecimento do arame se dá fundamentalmente por efeito Joule. Como consequência, a taxa de fusão se torna ainda menor. Mas ao ter a gota destacada, o arco abre novamente (dadas as condições favoráveis como energia térmica residual suficiente para reemissão de elétrons [16]) numa condição de corrente alta, o que faz com que a taxa de fusão instantânea seja muito alta e maior do que a sua taxa de alimentação, facilitando o afastamento da ponta do arame da poça. Porém, após o curto, já com o arco reaberto, a corrente começa decair continuamente, 
progressivamente diminuindo a taxa de fusão instantânea. Essa taxa de fusão continua diminuindo até que seja menor do que a taxa de alimentação, quando novo curto acontece.

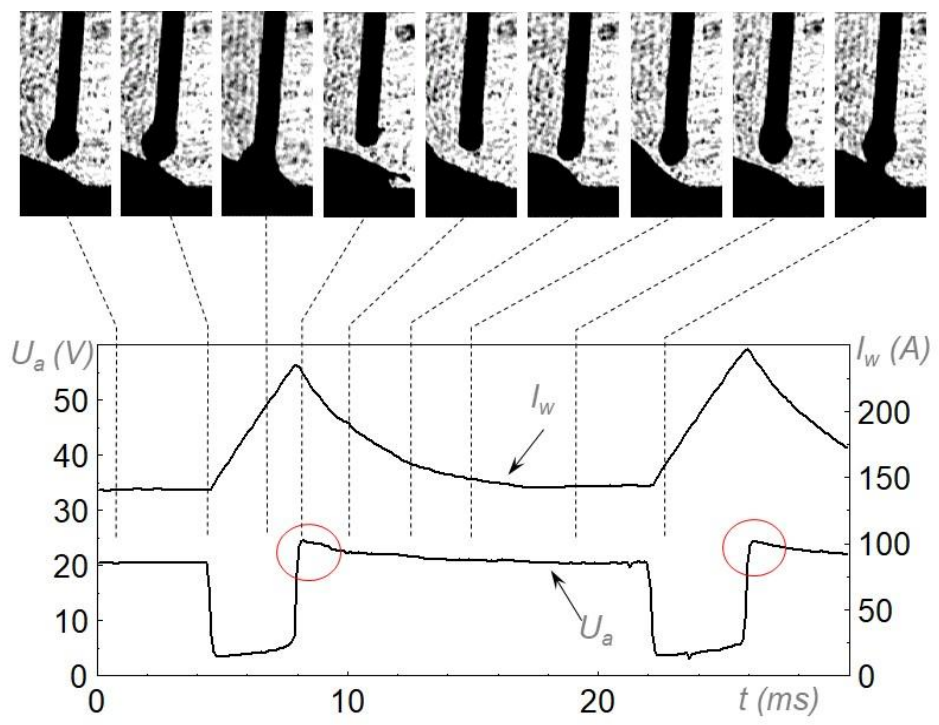

Figura 1. Oscilogramas típicos de corrente de soldagem de soldagem $\left(I_{w}\right)$ e tensão do arco $\left(U_{a}\right)$ durante a soldagem GMAW com transferência por curto circuito, com ilustração das gotas em transferência em fase com os transientes de corrente e tensão (os picos de tensão, destacados no oscilograma de tensão por círculos, são os chamados picos de reignição). Fonte: adaptada de [16].

Chama-se atenção para o instante de reabertura do arco no rompimento da ponte metálica, após cada curto-circuito. Devido à condição energética menos favorável ao final do curto-circuito (arco apagado), tem-se que a tensão requerida para se garantir a ionização do meio é maior do que a para manter o arco aceso. Essa maior tensão, em relação à tensão de arco aberto, varia de valor a cada curto-circuito em função das condições energéticas observadas para cada curto. Por isto, como destacado na Figura 1, existe a cada início de ciclo um pulso de tensão (não imposto, mas demandado) denominado de picos de reignição. A tensão do pico de reignição, pelo seu caráter, é menor do que a tensão de abertura do arco, já que na reabertura já há mais calor no sistema do que nas aberturas.

A metodologia proposta assume como válida a premissa de que uma maior regularidade da distribuição de frequências de picos de reignição de um arco é consequência da seleção de parâmetros de soldagem que produzem arcos de alta estabilidade. Portanto, uma seleção correta de parâmetros de soldagem leva a uma alta regularidade de distribuição de picos de tensão. Assim, para assistir esta metodologia, foi desenvolvido um algoritmo em Matlab@ para contagem e classificação dos picos de reignição de tensão de diferentes soldagens. O algoritmo desenvolvido organiza e registra a frequência dos picos de tensão observados em categorias de $1 \mathrm{~V}$, na forma de um vetor. As amostras de picos de tensão são coletadas de um sinal de tensão de soldagem mediante a captura de 150.000 valores obtidos de uma faixa do sinal (30 s de soldagem com taxa de amostragem de $5 \mathrm{kHz}$ ), que exclui a abertura e extinção do arco de soldagem.

O primeiro desafio foi definir qual é o limite inferior da faixa de ocorrência de picos de reignição de um arco com transferência por curto-circuito. Arbitrariamente, definiu-se como pico de reignição qualquer valor de tensão que esteja acima

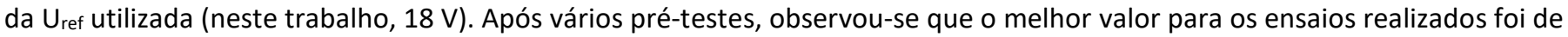
$22 \mathrm{~V}$, sendo este o valor tomado como limite inferior. Já o limite superior foi definido como $33 \mathrm{~V}$, visto que a ocorrência de picos de tensão acima deste valor apresenta incidências de menos de cinco observações, o que invalida o teste [18]. A Figura 2 mostra o domínio de tensões de pico analisado. O vetor picos de tensão é utilizado para o cálculo do parâmetro $\chi^{2}$ (explicado a seguir) e a apresentação dos resultados é feita através do programa de análise estatística Minitab@. 


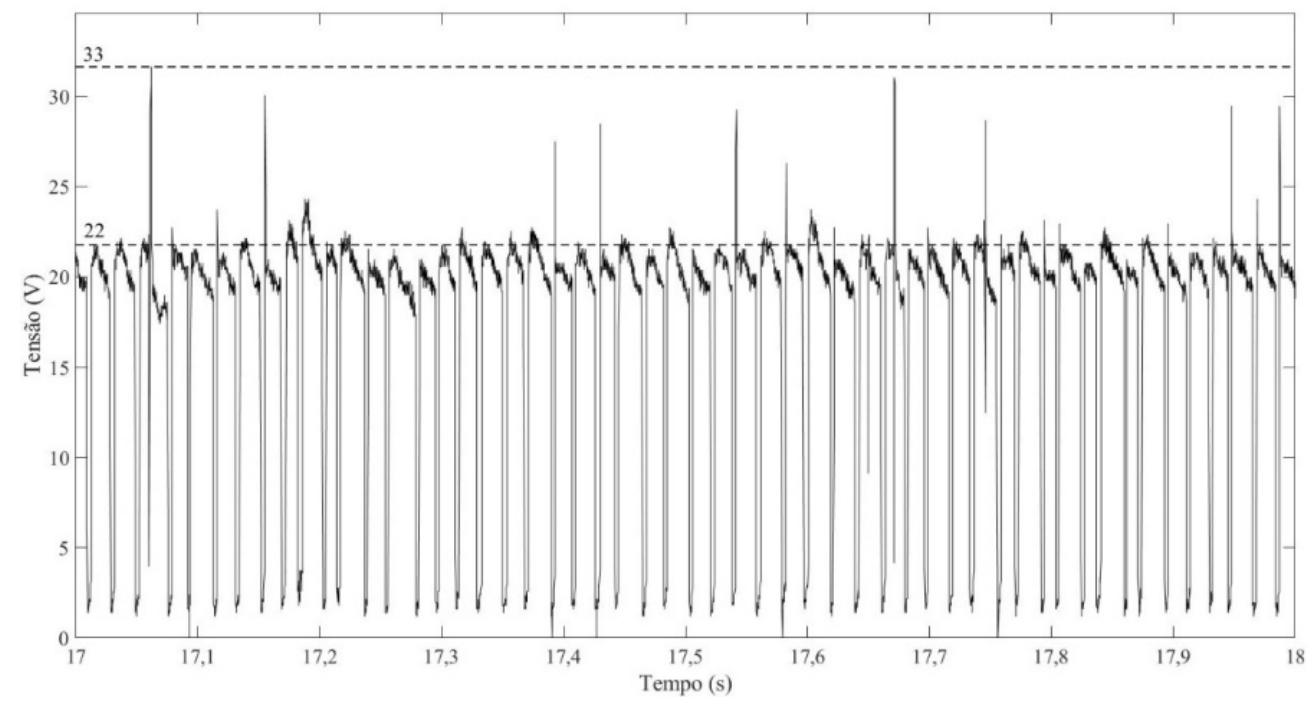

Figura 2. Oscilograma de tensão com indicação de faixa de ocorrência de picos de tensão de reignição (22 a 33 V) de um arco GMAW-CC com gás de proteção $\mathrm{Ar}+20 \% \mathrm{CO}_{2}$.

\subsection{Determinação da distribuição hipotética esperada de picos de tensão de reignição para uma condição padrão de soldagem}

A distribuição esperada é a probabilidade hipotética $P_{i}$, sendo que $P_{i}$ é a provável distribuição (esperada) de picos de tensão em diferentes faixas de tensão (categorias) para qualquer outro cordão de solda que venha a ser executado com os mesmos parâmetros de soldagem, incluindo arame e gás. Assim, a probabilidade hipotética $P_{i}$ de cada categoria $i$ é dada pela Equation 1.

$P_{i}=\frac{O_{i}^{\prime}}{n^{\prime}} \cdot 100$

onde $O_{i}^{\prime}$ é a frequência observada da i-ésima categoria da solda padrão e $n^{\prime}$ o tamanho da amostra.

Para demonstrar na prática a metodologia proposta para determinação da distribuição hipotética esperada de picos de tensão de reignição para uma condição padrão de soldagem, foram adotados, inicialmente, os parâmetros fixos de soldagem mostrados na Tabela $1 \mathrm{com}$ a proteção pela mistura $\mathrm{Ar}+20 \% \mathrm{CO}_{2}$, a um fluxo de $16 \mathrm{l} / \mathrm{min}$. Estes valores estão dentro de um domínio de parâmetros comumente adotados em soldagem GMAW-CC. Onze categorias de $1 \mathrm{~V}$ de extensão foram definidas entre os a valores arbitrários de 22 a $33 \mathrm{~V}$ (Figura 2). A distribuição esperada de picos de tensão foi obtida de uma primeira solda executada nas condições apontadas. Assim, a probabilidade hipotética $P_{i}$ de cada uma das categorias $i$ foi calculada pela Equation 1 e está mostrada na Tabela 2.

Essa probabilidade hipotética $P_{i}$ passa a ser aceita depois de testada. Para tanto, estabeleceu-se as seguintes hipóteses nula e alternativa:

$\mathrm{H}_{0}$ : a distribuição esperada de picos de tensão de reignição do arco para as condições de soldagem da Tabela 1 e gás de proteção $\mathrm{Ar}+20 \% \mathrm{CO}_{2}$ a $16 \mathrm{l} / \mathrm{min}$ é $P_{i}$

$\mathrm{H}_{1}$ : a distribuição de picos de tensão de reignição do arco nas condições de soldagem da Tabela 1 e gás de proteção $\mathrm{Ar}+20 \% \mathrm{CO}_{2}$ a $16 \mathrm{l} / \mathrm{min}$ difere de $P_{i}$.

\subsection{Teste de hipótese qui-quadrado}

O teste de hipótese é feito executando uma repetição do cordão de solda (mesmas condições de soldagem do cordão original). A repetição do cordão de solda forneceu o número total ( $\left.n^{\prime \prime}\right)$ de picos de tensão observados entre $22 \mathrm{~V}$ e $33 \mathrm{~V}$ de 1.311, que novamente foram classificados em 11 categorias. Neste segundo cordão, calcula-se a frequência esperada $E_{i}$ através da Equation 2.

$E_{i}=\frac{n^{\prime \prime} \cdot P_{i}}{100}$

A estatística do teste qui-quadrado é calculada pela Equation 3:

$\chi^{2}=\sum_{i=1}^{k} \frac{\left(O_{i-} E_{i}\right)^{2}}{E_{i}}$

onde $k$ é o número total de categorias (onze) e $O_{i}$ é a frequência observada da i-ésima categoria da repetição do cordão de solda. 
A metodologia proposta tem ampla aplicação na determinação da qualidade de ajuste de modelos. Neste trabalho, ela permitiu o estudo e comparação da distribuição de picos de reignição de soldagem entre dois arcos.

Para testar a hipótese, neste trabalho considerou-se um nível de significância $\alpha=0,05$. 0 valor crítico $\chi_{\text {crit. }}^{2}$ para 10 graus de liberdade (tabelado) é 18,3 [18]. A plataforma computacional utilizada para o cálculo da estatística $\chi^{2}$ foi o Minitab ${ }^{\odot}$, fornecendo o valor de 13,4. Portanto, não há evidência suficiente, ao nível de significância de $5 \%$, para rejeição da hipótese nula. Desta forma, conclui-se que $P_{i}$ é a distribuição esperada dos picos de tensão para as condições da Tabela 1 e gás $A r+20 \%$ $\mathrm{CO}_{2}$ a $16 \mathrm{I} / \mathrm{min}$.

A Tabela 2 mostra, além da distribuição hipotética $P_{i}$ (que foi testada) obtida do cordão original, a frequência dos picos de tensão esperados $E_{i}$ e a frequência observada $O_{i}$ em cada categoria para a solda de repetição. $O$ teste exige que as frequências esperadas não podem conter valores menores que 5 enquanto as frequências observadas devem ser obtidas de amostras aleatórias [18].

Tabela 2. Distribuição hipotética $P_{i}$ dos picos de tensão de reignição de arco para $\mathrm{Ar}+20 \% \mathrm{CO}_{2}$ a $16 \mathrm{I} / \mathrm{min}$ obtida do primeiro cordão de solda (condição padrão) e frequências observadas $O_{i}$ e esperadas $E_{i}$ do cordão de repetição.

\begin{tabular}{|c|c|c|c|}
\hline Categoria $i(V)$ & $P_{i}(\%)$ & $O_{i}$ & $E_{i}$ \\
\hline $22-23$ & 27,7 & 351 & 363,1 \\
\hline $23-24$ & 15,5 & 198 & 203,2 \\
\hline $24-25$ & 11,3 & 143 & 148,1 \\
\hline $25-26$ & 8,8 & 105 & 115,4 \\
\hline $26-27$ & 7,2 & 84 & 94,4 \\
\hline $27-28$ & 7,2 & 96 & 94,4 \\
\hline $28-29$ & 6,1 & 86 & 79,9 \\
\hline $29-30$ & 6,5 & 105 & 85,2 \\
\hline $30-31$ & 5,8 & 82 & 76,0 \\
\hline $31-32$ & 2,8 & 49 & 36,7 \\
\hline $32-33$ & 1,2 & 12 & 15,7 \\
\hline Total & 100 & 1.311 & \\
\hline
\end{tabular}

\section{Resultados e Discussões}

\subsection{Distribuição dos picos de tensão de reignição de arco para mesmas condições de soldagem}

Como já testado na seção $3, P_{i}$ (Tabela 2) é a distribuição esperada para qualquer outro cordão de solda que seja produzido nas condições da Tabela 1 e gás de proteção $\mathrm{Ar}+20 \% \mathrm{CO}_{2}$ a $16 \mathrm{l} / \mathrm{min}$. Isso significa que a distribuição de tensão de reignição de arco do segundo cordão obedece à distribuição $P_{i}$. Desta forma, conclui-se que quaisquer outros cordões de solda produzidos segundo as condições de soldagem de $P_{i}$ apresentarão distribuições de picos de tensão estatisticamente iguais, a um nível de confiança de $95 \%$, desde que realizados naquelas condições. A repetição do cordão gerou os resultados também mostrados na Tabela 2 e analisados através da Figura 3.

A Figura 3a mostra as frequências observadas e esperadas em suas categorias. Visualmente, existe uma distribuição de tensões bastante consistente, onde não se pode descartar a hipótese $\mathrm{H}_{0}$ como já visto na seção 3 . A Figura 3 b mostra que os intervalos de tensão que mais contribuíram para o aumento do valor de $\chi^{2}$ foram as categorias 29-30 e 31-32 V. Isso quer dizer que essas categorias apresentaram as maiores diferenças entre os valores observados e esperados, contribuindo desfavoravelmente para a não rejeição da hipótese nula. Essas categorias são picos de tensão de mais alto valor, entre 29 e $32 \mathrm{~V}$, mais próximos do limite superior. 


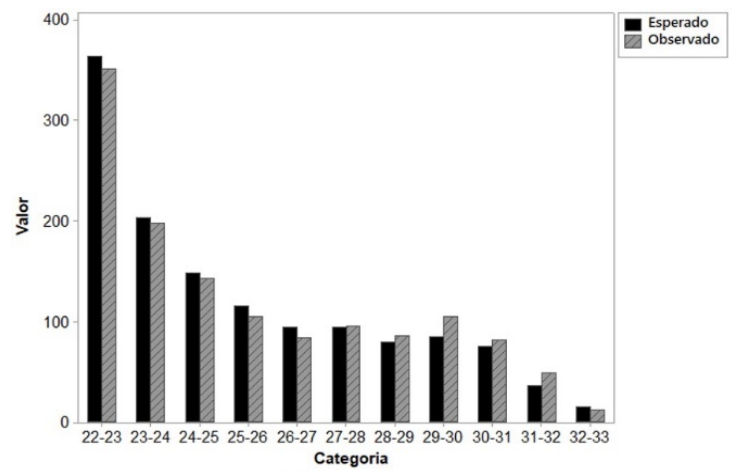

(a)

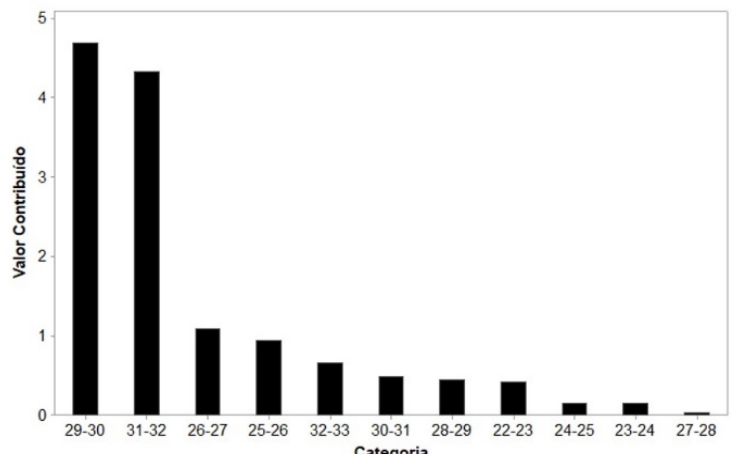

(b)

Figura 3. Resultados da metodologia proposta aplicada a cordão de solda produzido sob as condições da Tabela 1 e gás $\mathrm{Ar}+20 \% \mathrm{CO}_{2}$ a 16 I/min: (a) carta de valores observados e esperados, (b) contribuições para o valor $\chi^{2}$ por categoria.

A mesma metodologia foi aplicada para cordão de solda produzido de acordo com a Tabela 1, 16 I/min, mas, agora, o gás de proteção é o argônio puro. Os resultados estão na Figura 4. Novamente, existe uma distribuição visual de tensões de arco bastante consistente (Figura 4a). Para este gás, a probabilidade hipotética $P_{i}^{A r}$ (\%) encontrada foi (em valores crescentes de categorias de tensão): $P_{i}^{A r}=(17,0 ; 12,4 ; 11,2 ; 8,1 ; 8,8 ; 10,0 ; 12,4 ; 12,9 ; 5,8 ; 1,4 ; 0,13)$. Para esta $P_{i}^{A r}$, a hipótese nula não pode ser rejeitada diante de uma estatística $\chi^{2}$ de 14,6 . A Figura $4 \mathrm{~b}$ mostra que os intervalos de tensão que mais contribuíram para o aumento da estatística $\chi^{2}$ foram as classes 31-32 e 24-25 V, seguidos imediatamente por 30-31 e 32-33 V.

A metodologia desenvolvida e mostrada nos itens 3.1 e 3.2 e aplicada nas condições de soldagem descritas acima permitiu encontrar uma distribuição esperada de picos de tensão de reignição de arco $P_{i}$ para o gás $\mathrm{Ar}+20 \% \mathrm{CO}_{2}$ e $P_{i}^{A r}$ para o argônio puro. O teste de hipótese $\chi^{2}$ mostrou que as distribuições $P_{i}$ e $P_{i}^{A r}$ são as distribuições esperadas, a um nível de confiança de $95 \%$, para qualquer repetição do cordão de solda nas mesmas condições respectivas de soldagem. Portanto, pode-se afirmar que arcos produzidos pelos parâmetros de soldagem mostrados na Tabela 1, a um fluxo de gás de $16 \mathrm{l} / \mathrm{min}$, são arcos de soldagem estatisticamente iguais no que diz respeito à reignição do arco.

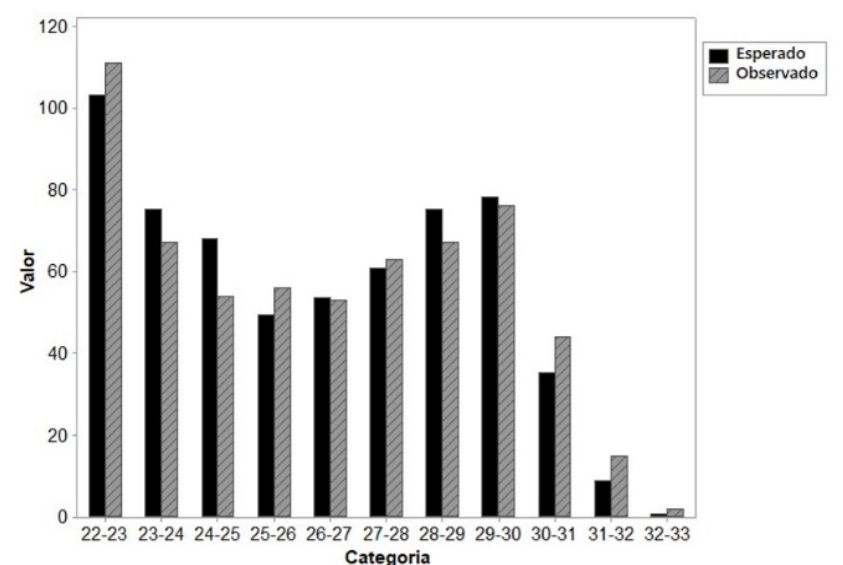

(a)

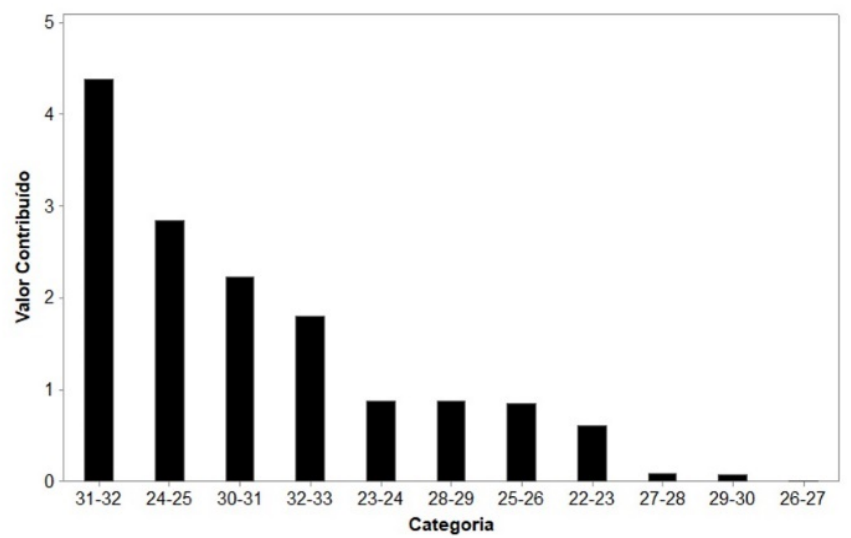

(b)

Figura 4. Resultados da metodologia proposta aplicada a cordão de solda produzido sob as condições da Tabela 1 e gás argônio puro a $16 \mathrm{l} / \mathrm{min}$ : (a) carta de valores observados e esperados, (b) contribuições para o valor qui-quadrado por categoria.

Uma terceira condição de soldagem também foi testada utilizando a metodologia proposta (itens 3.1 e 3.2 ), nas condições da Tabela 1 , a $16 \mathrm{l} / \mathrm{min}$, mas com gás $\mathrm{CO}_{2}$ puro. Para este gás, a probabilidade hipotética $P_{i}^{\mathrm{CO} 2}$ (\%) encontrada foi (em valores crescentes de categorias de tensão): $P_{i}^{C O 2}=(17,3 ; 22,0 ; 20,3 ; 13,3 ; 8,9 ; 6,9 ; 5,6 ; 3,9 ; 1,4 ; 0,4 ; 0,1)$. Para esta $P_{i}^{C O 2}$, a hipótese nula foi rejeitada para uma estatística $\chi^{2}$ de 2.847 . Assim, pode-se afirmar que, para $\mathrm{CO}_{2}$ puro, $\mathrm{cordões}^{\mathrm{C}}$ de solda produzidos nas mesmas condições de soldagem apresentarão distribuições de picos de tensão diferentes de $P_{i}^{C O 2}$. A Figura 5a mostra consistência na comparação visual, mas havendo rejeição da hipótese nula. $\mathrm{Como} \mathrm{sabido}^{19}$ ], $\mathrm{CO}_{2}$ puro como gás de proteção demanda maior tensão para se manter um arco de mesmo comprimento do que uma mistura de $\mathrm{CO}_{2}$ com argônio. Assim, esse resultado pode ser explicado pela diminuição do comprimento do arco (proporcionado pela troca de gás para $\mathrm{CO}_{2}$ ) e que resultou em um comprimento de arco muito pequeno levando a instabilidades na transferência metálica e maiores picos de reignição. Observa-se também que os maiores valores de contribuição para o $\chi^{2}$ estão nas categorias de tensão mais elevada 32-33 e 31-32 V (Figura 5b). 


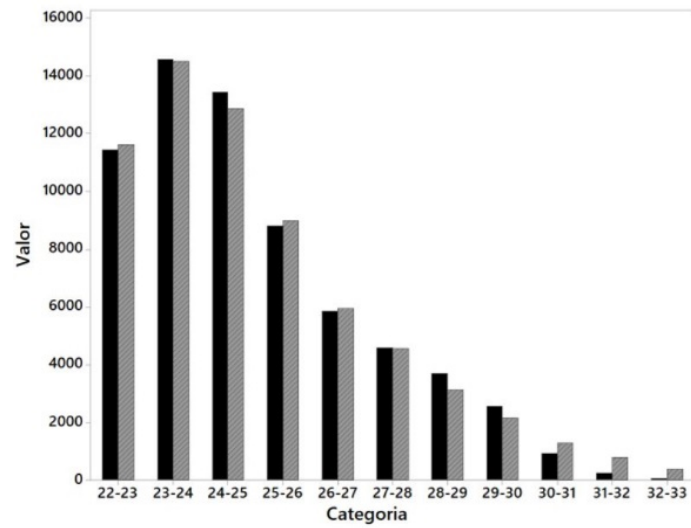

(a)

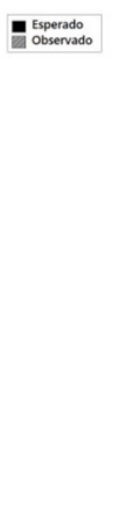

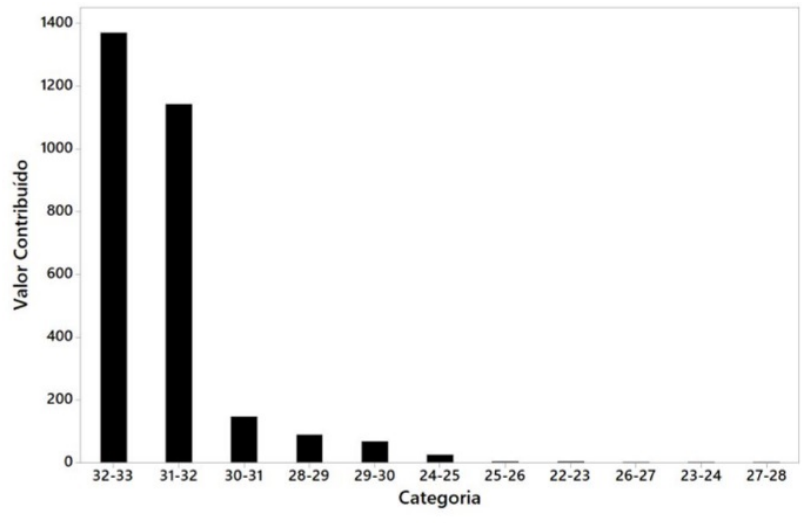

(b)

Figura 5. Resultados da metodologia proposta aplicada a cordão de solda produzido sob as condições da Tabela 1 e gás $\mathrm{CO}_{2}$ puro a $16 \mathrm{I} / \mathrm{min}$ : (a) carta de valores observados e esperados, (b) contribuições para o valor qui-quadrado por categoria.

\subsection{Distribuição dos picos de tensão de reignição de arco para diferentes condições de soldagem}

Com a intenção de avaliar a metodologia aplicada a diferentes condições de soldagem, foi testada a hipótese de o gás argônio puro apresentar a mesma distribuição de picos de tensão $P_{i}$ da mistura $\mathrm{Ar}+20 \% \mathrm{CO}_{2}$, ambos a $16 \mathrm{l} / \mathrm{min}$ e condições de soldagem da Tabela 1. Neste caso, a hipótese nula foi rejeitada com uma estatística $\chi^{2}$ de 78,9 . Pode-se dizer, então, que os arcos elétricos com mesmas regulagens, mas com dois diferentes gases de proteção, se comportam de maneiras diferentes com relação à reignição. É um resultado esperado exatamente pelo fato de os dois gases serem diferentes, indicando que a metodologia é coerente. A Figura 6 mostras os resultados de distribuição.

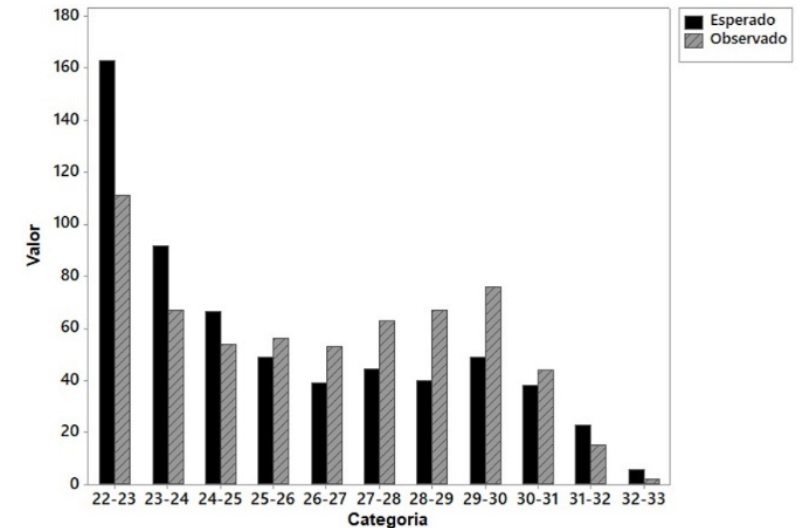

(a)

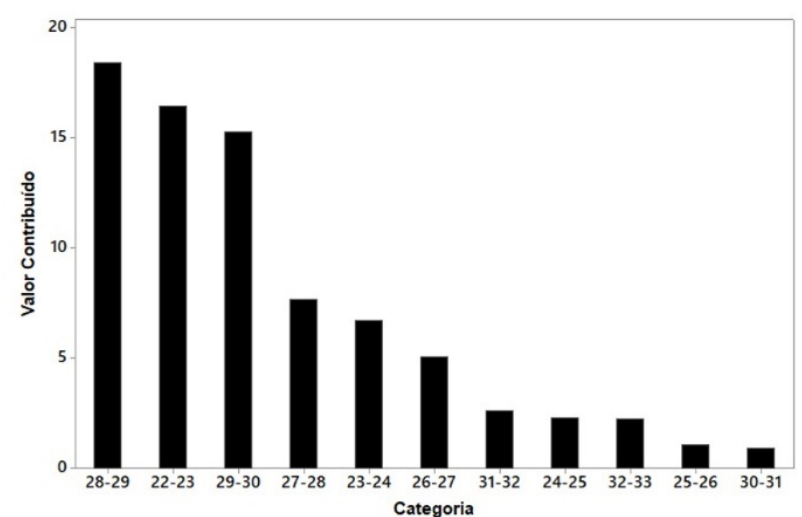

(b)

Figura 6. Resultados da metodologia proposta aplicada à comparação entre dois cordões de solda produzidos sob as condições da Tabela $1 \mathrm{e}$ gás argônio puro comparado à mistura $\mathrm{Ar}+20 \% \mathrm{CO}_{2}$, todos a $16 \mathrm{l} / \mathrm{min}$ : (a) carta de valores observados e esperados, (b) contribuições para o valor qui-quadrado por categoria.

Outra avaliação feita foi testar a hipótese de que uma solda realizada com a mistura $\mathrm{Ar}+20 \% \mathrm{CO}_{2}$, mas a um fluxo mais baixo de $10 \mathrm{l} / \mathrm{min}$, seguiria a distribuição de picos de tensão $P_{i}$. Nesta condição houve rejeição da hipótese nula. Foi um resultado inesperado, visto que a diminuição do fluxo de 16 para $10 \mathrm{l} / \mathrm{min}$ ainda está dentro das condições comumente aplicadas em soldagem, sendo, inclusive, vazão de gás considerada uma variável não-essencial em normas de soldagem. Os mesmos resultados de rejeição da hipótese nula foram encontrados para testes com gás argônio puro e diferentes fluxos de gás.

O fato da rejeição da hipótese nula para $\mathrm{CO}_{2}$ puro nas mesmas condições de soldagem (seção 4.1) somado ao fato de se ter rejeição da hipótese nula para fluxo de gases diferentes são indícios de que não é qualquer combinação de parâmetros de soldagem, mesmo sendo parâmetros normalmente aplicados e amplamente aceitos, que produz arcos de soldagem com características de alta regularidade dos picos de reignição. É provável que exista uma combinação particular de parâmetros que promova destacada regularidade de picos de tensão. Como encontrar esta combinação, mostrar como e o porquê ela conduz a um arco de alta regularidade são assuntos que devem ser investigados em trabalhos futuros. Ainda em trabalhos futuros, cabe avaliar a execução da metodologia proposta para taxas de aquisição maiores que $5 \mathrm{kHz}$, dado que os picos de reignição são eventos de duração muito curta. 


\subsection{Avaliação da estabilidade do arco pelo Índice Vilarinho (IVcc)}

A estabilidade do arco elétrico pode ser avaliada por vários métodos. Será tomada aqui a avaliação de estabilidade desenvolvida por Rezende et al. [20] através da adoção do Índice Vilarinho (IVCC) como indicador de estabilidade, sendo definido pela equation 4 :

$I V_{c c}=\frac{\sigma_{t s c}}{t_{s c}}+\frac{\sigma_{t o a}}{t_{o a}}$

onde $\sigma_{t s c}$ e $\sigma_{t o a}$ definem o desvio padrão do tempo de curto circuito e do tempo de arco aberto, respectivamente, e $t_{s c}$ e $t_{o c}$ são as médias de tempo de curto circuito e de arco aberto.

Quanto menor o índice, mais estável (regular) é um arco elétrico. O IVcc foi calculado para o argônio puro, a mistura $\mathrm{Ar}+20 \% \mathrm{CO}_{2}$ e suas repetições; para fluxo de $16 \mathrm{l} / \mathrm{min}$. O valor médio de IVcc para a mistura foi de 0,37 enquanto para argônio puro de 0,33. Esses valores de IVcc são da ordem de grandeza dos menores valores encontrados na literatura consultada. Parece haver uma relação entre regularidade do arco elétrico (expresso pelo IVcc) e a regularidade da reignição do arco. Quanto mais regular um arco como um todo, mais regular também são os picos de reignição. No entanto, mais estudos devem ser dedicados no intuito de confirmar essa possível relação. Mas, novamente, parece existir uma combinação de parâmetros de soldagem capaz de oferecer máxima estabilidade (regularidade) dos picos de tensão de reiginição do arco de soldagem e isso pode estar ligado à máxima regularidade do sinal de tensão como um todo.

\section{Conclusões}

A metodologia proposta permitiu o estudo estatístico da distribuição de picos de reignição de arcos de soldagem. Foi demonstrada haver potencialmente uma outra forma de avaliar a estabilidade de arcos em soldagem GMAW-CC, ou seja, através da análise de distribuição de picos de reignição através do teste $\chi^{2}$ de ajuste de modelo. Os resultados do teste aplicado neste trabalho permitem concluir que existe uma distribuição esperada de picos de tensão de reignição de arco, para os gases $\mathrm{Ar}+20 \% \mathrm{CO}_{2}$ e argônio puro, a um dado nível de confiança estatística.

\section{Agradecimentos}

Os autores agradecem à Faculdade de Engenharia da UFJF, através do Laboratório para Otimização de Processos de Soldagem, pela utilização dos equipamentos e consumíveis. Agradecem também ao programa de Iniciação Científica - BIC da UFJF pela concessão de bolsas de Iniciação Científica.

\section{Referências}

[1] Suban M, Tusek J. Methods for the determination of arc stability. Journal of Materials Processing Technology. 2003;143-144:430-437. http://dx.doi.org/10.1016/S0924-0136(03)00416-3.

[2] Roca AS, Fals HC, Fernández JB, Macías EJ, Adán FS. New stability index for short circuit transfer mode in GMAW process using acoustic emission signals. Science and Technology of Welding and Joining. 2007;12(5):460-466. http://dx.doi.org/10.1179/174329307X213882.

[3] Simpson SW. Signature images for arc welding fault detection. Science and Technology of Welding and Joining. 2007;12(6):481-486. http://dx.doi.org/10.1179/174329307X213909.

[4] Huang $Y$, Wang K, Zhou Z, Zhou X, Fang J. Stability evaluation of short-circuiting gas metal arc welding based on ensemble empirical mode decomposition. Measurement Science and Technology. 2017;28(3):1-12.

[5] Zhiyong L, Qiang Z, Yan L, Xiaocheng Y, Srivatsan TS. An analysis of gas metal arc welding using the lyapunov exponent. Materials and Manufacturing Processes. 2013;28(2):213-219. http://dx.doi.org/10.1080/10426914.2012.746705.

[6] Absi Alfaro SC, Carvalho GC, Cunha FR. A statistical approach for monitoring stochastic welding processes. Journal of Materials Processing Technology. 2006;175(1-3):4-14. http://dx.doi.org/10.1016/j.jmatprotec.2005.04.049.

[7] Adolfsson S, Bahrami A, Bolmsjo G, Claesson I. On-line quality monitoring in short-circuit gas metal arc welding. Welding Journal. 1999;78:59s-73s.

[8] Quinn TP, Smith C, McCowan CN, Blachowiak E, Madigan RB. Arc sensing for defects in constant-voltage gas metal arc welding. Welding Journal. 1999;78(9):322s-328s.

[9] Liu S, Siewert TA. Metal transfer in gas metal arc welding: Droplet rate. Welding Journal. 1989;68:52s-58s.

[10] Chen M, Zhang D, Wu C. Current waveform effects on CMT welding of mild steel. Journal of Materials Processing Technology. 2017;243:395-404. http://dx.doi.org/10.1016/j.jmatprotec.2017.01.004.

[11] Hermans MJM, Ouden GD. Process behavior and stability in short circuit gas metal arc welding. Welding Journal. 1999;78:137s-141s. 
[12] Chu YX, Hu SJ, Hou WK, Wang PC, Marin SP. Signature analysis for quality monitoring in short-circuit GMAW. Welding Journal. 2004;83(12):336s-343s.

[13] Souza D, Rossi ML, Keocheguerians F, Nascimento VC, Vilarinho LO, Scotti A. Influência da Regulagem de Parâmetros de Soldagem sobre a Estabilidade do Processo MIG/MAG Operando em Curto-Circuito. Soldagem e Inspeção. 2011;16(1):22-32. http://dx.doi.org/10.1590/S0104-92242011000100004.

[14] Kang MJ, Rhee S. The statistical models for estimating the amount of spatter in the short circuit transfer mode of GMAW. Welding Journal. 2001;80(1):1s-8s.

[15] Zhu, Z., Wu, W. and Chen, Q. Random nature of droplet size and its origins in short circuit $\mathrm{CO}_{2}$ arc welding. Science and Technology of Welding and Joining. 2005;10(6):636-642.

[16] Scotti A, Ponomarev V. Soldagem MIG/MAG-melhor entendimento melhor desempenho. São Paulo: Ed. Artliber; 2014.288 p.

[17] Scotti A, Gomes M, Pereira J. Use assessment of electronic power sources for SMAW. Revista de Metalurgia. 1999;35(2):84-90. http://dx.doi.org/10.3989/revmetalm.1999.v35.i2.610.

[18] Larson R, Farber B. Estatística aplicada. 6. ed. São Paulo: Pearson editora; 2015. 656 p.

[19] Liskévych O, Scotti A. Influence of the $\mathrm{CO}_{2}$ Content on Operational Performance of Short-Circuit GMAW. Welding in the World. 2014;59(2):217-224. http://dx.doi.org/10.1007/s40194-014-0196-x.

[20] Rezende GMC, Liskévych O, Vilarinho LO, Scotti A. Um Critério para Determinar a Regulagem da Tensão em Soldagem MIG/MAG por Curto-Circuito. Soldagem e Inspeção. 2011;16(2):98-103. http://dx.doi.org/10.1590/S0104-92242011000200002. 